\title{
High-throughput analysis of DNA interstrand crosslinks in human peripheral blood mononuclear cells by automated reverse FADU assay
}

\author{
Małgorzata Dębiak $^{\mathrm{a}}$, Alicja Panas ${ }^{\mathrm{a}, 1}$, Dirk Steinritz $^{\mathrm{b}}$, Kai Kehe ${ }^{\mathrm{b}}$, Alexander Bürkle $^{\mathrm{a}, *}$ \\ a Molecular Toxicology Group, Department of Biology, Box X911, University of Konstanz, D-78457 Konstanz, Germany \\ ${ }^{\mathrm{b}}$ Bundeswehr Institute of Pharmacology and Toxicology, Neuherbergstr. 11, D-80937 Munich, Germany
}

Keywords:

DNA interstrand crosslinks

FADU assay

Comet assay

Sulphur mustard

Melphalan

\begin{abstract}
A B S T R A C T
DNA interstrand crosslinks (ICL) are induced both by several cytotoxic anti-cancer drugs as well as by the chemical warfare agent sulphur mustard (SM). Although measurement of ICL formation could be used in risk assessment or provide valuable predictive information on the response of malignant cells to crosslinking chemotherapeutic agents, respectively, it is currently not applied due to lack of appropriate standardized methodology. Here we describe a fast and convenient procedure for detection of ICL in human peripheral blood mononuclear cells (PBMC) as high-throughput method, termed 'reverse FADU assay'. This assay detects ICL based on the prevention of time-dependent alkaline unwinding of double-stranded DNA in a cell lysate that starts from single or double strand breaks. We have successfully established and optimized the reverse FADU assay by using human PBMC exposed to the model compounds mitomycin C, melphalan and SM. Our fully automated assay version is faster than currently used methods and possesses similar sensitivity. It operates in a 96-well format, thus allowing parallel analysis of multiple samples. Furthermore, we describe optimized protocols for sample preparation, with sample volume minimized to $100 \mu \mathrm{l}$ of blood, storage and shipment conditions. We conclude that the reverse FADU assay is an attractive candidate method for monitoring DNA damage induced by DNA crosslinking agents.
\end{abstract}

\section{Introduction}

DNA crosslinking agents represent a structurally diverse group of compounds including sulphur and nitrogen mustard derivatives, nitrosoureas, and antibiotics such as mitomycin C (Lawley and Phillips, 1996; Noll et al., 2006). The critical lesion consists of a covalent bond between complementary DNA strands, which prevents strand separation leading to transcription and replication blockage and, in many cases, to cell death (Kee and D'Andrea, 2010; Noll et al., 2006; Dronkert and Kanaar, 2001; Lawley and Phillips, 1996). Due to their exquisite toxicity, DNA crosslinking agents have been used for decades as cytostatic drugs. The first crosslinking agent to be synthesized was SM, a chemical warfare agent that was first

\footnotetext{
* Corresponding author at: Molecular Toxicology Group, Department of Biology, Box X911, University of Konstanz, D-78457 Konstanz, Germany. Tel.: +497531 884035; fax: +497531884033.

E-mail addresses: Malgorzata.Debiak@uni-konstanz.de (M. Dębiak), Alicja.Panas@kit.edu (A. Panas), dirksteinritz@bundeswehr.org (D. Steinritz), KaiKehe@bundeswehr.org (K. Kehe), Alexander.Buerkle@uni-konstanz.de (A. Bürkle).

1 Present address: Institute of Toxicology and Genetics, Forschungszentrum Karlsruhe, Karlsruhe Institute of Technology, D-76344 Eggenstein-Leopoldshafen, Germany.
}

used during the World War I. Later on, controlled medical application of SM for cancer treatment was investigated but failed because of difficulties in handling and the high reactivity of the compound, which rendered systemic application impossible. However, in subsequent years less reactive derivatives of nitrogen mustard were synthesized. The agents induce formation of several different adducts within DNA, the majority of which being monoadducts resulting from of a single substitution event. A small proportion of monoadducts are engaged in a second reaction producing DNA crosslinks. Although interstrand crosslinks represent only a minority of DNA adducts, they are thought to be critical for cell death induction. Their formation, persistence and repair clearly correlate with cytotoxicity in vitro both in prokaryotic and eukaryotic cells (Noll et al., 2006; Dronkert and Kanaar, 2001; McHugh et al., 2001). Importantly, a positive correlation was found between patient survival and level of DNA crosslinks in vivo. After high-dose melphalan treatment patients with increased DNA adducts formation and slower repair showed improved response and progression-free survival (Souliotis et al., 2003). Furthermore, it was shown that ex vivo DNA damage in PBMC reflects the in vivo situation (Spanswick et al., 2002). Therefore the amount of DNA crosslinks assessed ex vivo in PBMC might be used as predictive biomarker for high-dose melphalan treatment in multiple myeloma patients (Dimopoulos et al., 2007). Additionally, in case of cyclophosphamide and ifosfamide, 
the evaluation of crosslink formation in PBMC of patients yields information on metabolic drug activation. Both are administered as inactive pro-drugs and undergo metabolic activation in the liver resulting in the formation of reactive metabolites, i.e. 4-hydroxycyclophosphamide and 4-hydroxy-ifosfamide. The monitoring of crosslink formation could therefore serve as an indicator for the effective dose reached in the patient (Hartley et al., 1999).

Although the assessment of DNA crosslinks might be highly beneficial for modern, individualized therapy this issue has been addressed only in a very limited number of studies. One of the reasons might be the lack of established, suitable methodology. So far, measurement of DNA crosslinks has been performed by alkaline filter elution, agarose gel based methods or reverse comet assay (Pfuhler and Wolf, 1996; Souliotis et al., 2003). While the first two require a large number of cells, they are not easily adapted for in vivo studies and may be problematic regarding patients' health and ethical considerations. The most suitable assay for this purpose appeared to be the reverse comet assay, due to the modest requirements for biological material. However, the standardization of comet assay is not an easy task due to problems with objectivity during evaluation. Variation in sample analysis was observed within one sample evaluated by different laboratory staff (Forchhammer et al., 2008). Consequently, despite years of investigation, only the alkaline version of the comet assay was recognised by the Organisation for Economic Co-operation and Development (OECD) and the appropriate guideline is in preparation (BrendlerSchwaab et al., 2005).

The aim of the present study was to develop a high-throughput, automated, standardized assay, operating on human PBMC for measurement of DNA crosslinks. The original version of the fluorimetric alkaline DNA unwinding assay (FADU assay) was described by Birnboim and Jevcak (1981) for detection of $\gamma$-ray induced DNA strand breaks in human PBMC. We have recently established an automated FADU for strand breaks detection. While its sensitivity of is comparable to the comet assay and alkaline filter elution, the simple and fast protocol and the robustness of the assay is an advantage (Moreno-Villanueva et al., 2009). The assay is based on the assumption that each strand break serves as an unwinding point during the alkaline denaturation of chromosomal DNA in the cell lysate. Thus at defined temperature and $\mathrm{pH}$ conditions the extent of DNA strand separation is dependent on number of strand breaks and can be standardized with respect to the initial level of induced strand breaks by use of ionizing radiation, which produces known numbers of DNA strand breaks per cell. The unwinding is quantified by means of a fluorescence dye that binds exclusively to the DNA that remains double stranded after completion of the alkali incubation. The manually operated version of the FADU assay was successfully applied for measurement of DNA strand breaks in different cell types and organelles upon irradiation and chemical treatment (Baumstark-Khan et al., 1992, 2000; Roos et al., 2000). Surprisingly, in contrast to the comet assay it has not been adopted by many laboratories. Our automated, microtiter-based assay format seems to be an ideal candidate for standardized, large-scale biomonitoring studies. Here, we describe the automated reverse FADU assay optimized for assessment of DNA interstrand crosslinks in human PBMC, including optimized procedures for preparation, storage and shipment of samples.

\section{Materials and methods}

\subsection{Isolation of peripheral blood mononuclear cells (PBMC)}

Peripheral venous blood was obtained from healthy volunteers and samples were anonymized. Ethical approval had been obtained by the University of Konstanz Ethics Committee. PBMC were isolated by Biocoll (Biochrom AG, Berlin, Germany) gradient centrifugation according to manufacturer's protocol. PBMC were maintained in RPMI 1640 medium (Invitrogen, Karlsruhe, Germany) supplemented with 10\% FCS (Biochrom AG, Berlin, Germany), $100 \mathrm{U} / \mathrm{ml}$ penicillin and $100 \mu \mathrm{g} / \mathrm{ml}$ streptomycin (Invitrogen, Karlsruhe, Germany) at a cell density of $10^{6}$ cells $/ \mathrm{ml}$ in a humidified atmosphere at $37{ }^{\circ} \mathrm{C}$ and $5 \% \mathrm{CO}_{2}$.

In order to stimulate T lymphocytes for proliferation, freshly isolated PBMC were incubated with $5 \mu \mathrm{g} / \mathrm{ml}$ PHA-L (Roche, Penzberg, Germany) for $48 \mathrm{~h}$ under standard culture conditions. The cell cycle distribution was analyzed by flow cytometry after staining with propidium iodide by using an established protocol (Roos et al., 2004). Forty-eight hours after addition of PHA-L the population reached asynchronous growth and all cell cycle phases were represented (data not shown). PHA-L was present in culture medium during the whole duration of the experiments, inclusive treatment with melphalan and post-incubation.

\subsection{Isolation of human leukocytes by erythrocyte lysis}

Isolation of leukocytes by erythrocyte lysis was performed by using an erythrocyte lysis kit (R\&D Systems, Minneapolis, MN) according to manufacturer's protocol.

\subsection{Cell treatment with DNA-crosslinking agents}

Mitomycin C, melphalan and CEES were purchased from Sigma-Aldrich GmbH (Daisenhofen, Germany). Mitomycin C was dissolved in water (final concentration $500 \mu \mathrm{g} / \mathrm{ml}$ ) and stored at $4{ }^{\circ} \mathrm{C}$ for up to 7 days. Melphalan solution $(10 \mathrm{mg} / \mathrm{ml}$ in $95 \% \mathrm{EtOH} / 0.5 \% \mathrm{HCl}$ ) was prepared directly before use. Mutagen treatment was performed by adding aliquots of drug serial dilutions to cells in complete culture medium. PBMC were incubated in a cell culture incubator for $60 \mathrm{~min}$, collected by centrifugation and the mutagen-containing medium was carefully removed. Cells were washed with PBS, resuspended in culture medium and cultured under standard conditions.

SM was purchased from TNO (Rijswijk, The Netherlands). SM was initially dissolved in ethanol and then diluted to final concentration in Eagle Minimal Essential Medium without foetal calf serum immediately prior to cell treatment. Mutagen treatment was performed by adding aliquots of drug serial dilutions to cells in Minimal Essential Medium without calf serum. PBMC were incubated in a safety hood at room temperature for $30 \mathrm{~min}$, collected by centrifugation and the mutagen-containing medium was carefully removed. Cells were washed with PBS, resuspended in culture medium and cultured under standard conditions.

\subsection{Cryo-preservation of cells}

PBMC $\left(10^{6}\right.$ cells/well $)$ were harvested by centrifugation $\left(260 \times \mathrm{g}, 5 \mathrm{~min}\right.$ at $\left.4^{\circ} \mathrm{C}\right)$ and resuspended in pre-chilled culture medium supplemented with $10 \%$ DMSO. Cells were immediately transferred into cryo-tubes placed on ice and frozen down slowly at $-80{ }^{\circ} \mathrm{C}$ in cryo-container filled with $100 \%$ 2-propanol. For long-term storage wells were transferred to liquid nitrogen the next day.

PBMC were thawed in a water bath at $37^{\circ} \mathrm{C}$ for $1 \mathrm{~min}$. The cell suspension was combined with $5 \mathrm{ml}$ culture medium and pelleted by centrifugation $(200 \times g, 5 \mathrm{~min}$ at $4 \mathrm{C})$. PBMC were washed with $5 \mathrm{ml}$ PBS and immediately analyzed by automated FADU assay.

\subsection{Automated FADU assay}

The protocol of the automated FADU has been published recently (Moreno-Villanueva et al., 2009). Briefly, PBMC were 
resuspended in suspension buffer ( $10 \mathrm{mM}$ Na-phosphate buffer, $\mathrm{pH}$ 7.4; $0.25 \mathrm{M}$ meso-inositol; $1 \mathrm{mM} \mathrm{MgCl}_{2}$ ) at the final concentration of $10^{5}$ cells $/ \mathrm{ml}$. Leukocytes derived from $100 \mu \mathrm{l}$ whole-blood samples were resuspended in $2 \mathrm{ml}$ of suspension buffer. For analysis of DNA strand breaks, $70 \mu \mathrm{l}$ cell suspension was transferred on ice into 96-well plates. For reverse FADU assay cells were additionally X-irradiated on ice with a dose of 25 Gy. The FADU assay is fully automated and all pippeting steps are performed by a liquid handling device Genesis RSP 100 (Tecan AG, Hombrechtikon; Switzerland). Seventy $\mu$ l lysis buffer (9 M Urea, $10 \mathrm{mM}$ $\mathrm{NaOH}, 2.5 \mathrm{mM} \mathrm{1,2-cyclohexanedinitrilotetraacetic} \mathrm{acid,} \mathrm{0.1 \%} \mathrm{SDS)}$ was added at rate of $150 \mu \mathrm{l} / \mathrm{s}$ and samples were incubated for $12 \mathrm{~min}$ at $0^{\circ} \mathrm{C}$. Pre-chilled alkaline buffer ( $42.5 \%$ lysis buffer, $0.2 \mathrm{M}$ $\mathrm{NaOH}$ ) was overlaid on top of the cell lysate at a rate of $10 \mu \mathrm{l} / \mathrm{s}$. Alkaline unwinding was performed at 30 " $\mathrm{C}$ for $60 \mathrm{~min}$, then $140 \mu \mathrm{l}$ neutralization buffer ( $1 \mathrm{M}$ glucose, $14 \mathrm{mM} \beta$-mercaptoethanol) was added at a rate of $200 \mu \mathrm{l} / \mathrm{s}$. Following incubation at $22^{\circ} \mathrm{C}$ for $30 \mathrm{~min}$, $156 \mu \mathrm{l}$ SybrGreen solution (1:8333 v/v in $\mathrm{H}_{2} \mathrm{O}$; MoBiTec, Göttingen, Germany) was added. Samples were mixed by automatic pipetting up and down. Fluorescence was measured in a 96-well-plate reader by excitation at $492 \mathrm{~nm}$ and emission at $520 \mathrm{~nm}$. For each sample an internal control of DNA content was incorporated without alkaline unwinding process. Here the unwinding was prevented by addition of neutralization buffer to samples prior to the addition of alkaline buffer.

The ratio of double-stranded DNA was calculated as follows:

Double-stranded DNA $=$ fluorescence of sample/fluorescence of sample without unwinding

fluorescence of untreated control/fluorescence of untreated control without unwinding

\subsection{Comet assay}

Cells were protected from sunlight during the whole experimental procedure in order to avoid additional DNA damage. After exposure of PBMC to various concentrations of melphalan or SM, viability of cells was determined by trypan blue exclusion and was found to be greater than $97 \%$. Twenty $\mu \mathrm{l}$ of the cell suspension was combined with $200 \mu \mathrm{l}$ low-melting agarose at a temperature of $37^{\circ} \mathrm{C} .100 \mu \mathrm{l}$ of this mixture was transferred to agarose-coated slides and embedded in another layer of low-melting agarose. For reversed comet assay $20 \mu \mathrm{l}$ of the cell suspension was incubated with $0.002 \% \mathrm{H}_{2} \mathrm{O}_{2}$ for $10 \mathrm{~min}$ at $4{ }^{\circ} \mathrm{C}$ and then combined with $200 \mu \mathrm{I}$ low-melting agarose and embedded on agarose coated slides. The slides were placed in cold $\left(4^{\circ} \mathrm{C}\right)$ lysis buffer for $60 \mathrm{~min}$. To unwind the DNA, the slides were placed in an alkali solution for $30 \mathrm{~min}$ and then subjected to electrophoresis $(1 \mathrm{~V} / \mathrm{cm}, 30 \mathrm{~min})$ in an alkaline buffer $(\mathrm{pH}>13)$. After rinsing in distilled water, they were fixed with methanol at $-20^{\circ} \mathrm{C}$ for $10 \mathrm{~min}$. Slides were stained with ethidium bromide and measurements were done by means of a fluorescence microscope and "Komet 5.5" software (Kinetic Imaging Ltd.). 50 cells per slide were analyzed, thus yielding 100 data sets for each concentration (double determination).

\subsection{Statistical analysis}

Data analysis was performed by means of GraphPad Prism 5.0 (GraphPad Software, Inc., La Jolla, CA) software.

\section{Results}

\subsection{Adaptation of FADU assay for detection of DNA interstrand crosslinks}

The FADU assay is based on the direct relationship between the extent of alkali-induced DNA unwinding and number of DNA strand breaks. The principle of reverse FADU assay is analogous to the reverse comet assay and assumes that the alkaline unwinding is blocked at the sites of covalent bond between complementary DNA strands, i.e. ICL. Indeed, the measurement of DNA crosslinks can be performed indirectly by administering high-dose irradiation in order to induce large numbers of DNA strand breaks, causing massive alkali unwinding. If crosslinks are present, however, the unwinding would be decreased in comparison to the cells treated with irradiation only. Based on this rationale, we set up a reverse FADU assay based on a previously optimized automated protocol designed for detection of DNA strand breaks in human PBMC upon ionizing radiation (Moreno-Villanueva et al., 2009). In preliminary studies to determine the optimal conditions of DNA unwinding and treatment with DNA break inducing agent, human PBMC were treated with melphalan for $24 \mathrm{~h}$ in a dose dependent manner. Melphalan belongs to the group of nitrogen mustard derived cytostatics, but in contrast to the more frequently used cyclophosphamide does not require metabolic activation (Colvin and Quong, 2002). Thus it is a perfect model substance for establishing the assay. The method of choice for DNA strand break induction was ionizing radiation. Using the protocol of Moreno-Villanueva and colleagues (Moreno-Villanueva et al., 2009) DNA strand breaks were detected after treatment of PBMC with melphalan for $24 \mathrm{~h}$ in the dose range $2.5-10 \mu \mathrm{g} / \mathrm{ml}$. Within the same dose range melphalan crosslinks were detected after irradiation with $25 \mathrm{~Gy}$ or higher

(data not shown). Lower irradiation doses had a negative impact on detection of melphalan crosslinks. Variation of DNA unwinding conditions, particularly temperature and time, did not improve the detection level for melphalan crosslinks (data not shown). Thus the reverse FADU protocol included X-irradiation with $25 \mathrm{~Gy}$ and alkaline unwinding at $30^{\circ} \mathrm{C}$ for $60 \mathrm{~min}$, as described in Section 2.5 .

\subsection{Determination of detection limit of FADU assay for different DNA crosslinking agents}

To validate the protocol for the reverse FADU assay and determine the detection limit for DNA crosslinks, DNA damage was measured upon pulse treatment with melphalan and two further compounds, i.e. mitomycin C and SM, in dose-dependent manner (Fig. 1). For each compound the time point of maximal crosslink induction within the cell was chosen, which was directly after treatment for mitomycin C or SM and $14 \mathrm{~h}$ after treatment with melphalan. The induction of crosslinks was manifested by an increase in double-stranded DNA after treatment with crosslinking agents in comparison to untreated but irradiated samples. In all cases, a dose-dependent induction of DNA crosslinks was observed (Fig. 1, left panel). The lowest dose after which DNA crosslinks were detected was $5 \mu \mathrm{g} / \mathrm{ml}$ mitomycin C, $5 \mu \mathrm{g} / \mathrm{ml}$ melphalan and $3 \mu$ M SM, respectively ( $P<0.001$; Dunnett's Multiple Comparison Post Test). Noteworthy are the extremely small standard deviations and high reproducibility of experiments as a result of automation.

To evaluate the specificity of the reversed FADU assay for DNA crosslinks, we used 2-chloroethyl ethyl sulphide (CEES), which is a monofunctional sulphur mustard analogue. In the dose range up to $500 \mu \mathrm{M}$ CEES the fluorescence signal was identical to the control. This confirms that the increase in fluorescence of samples treated with the three other compounds is due to their crosslinking activity.

The FADU assay was performed simultaneously as direct and reverse assay (to assess DNA strand breaks and DNA crosslinks, respectively) in order to obtain maximal information on induced 

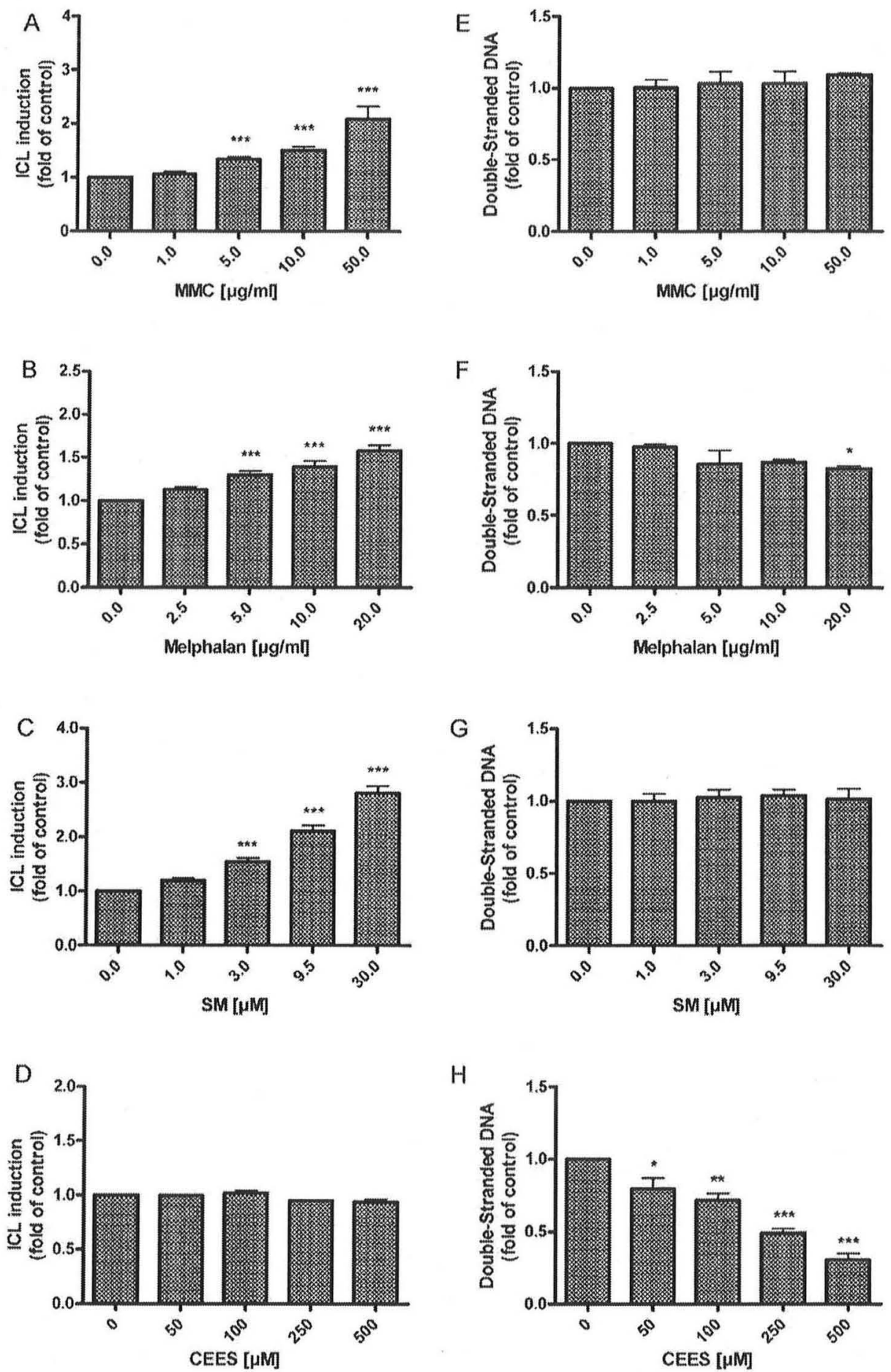

Fig. 1. Frequency of ICL and strand breaks in human PBMC after treatment with mitomycin C, melphalan, SM or CEES, as analyzed by automated FADU assay. PBMC were pulse-treated with mitomycin C (60 min), melphalan $(60 \mathrm{~min}), \mathrm{SM}(30 \mathrm{~min})$ or CEES $(60 \mathrm{~min})$. Mutagen-containing medium was removed by centrifugation, and cells were washed with PBS. Automated FADU assay was performed immediately after treatment with mitomycin C or CEES. After treatment with melphalan, cells were allowed to recover for $14 \mathrm{~h}$ in fresh culture medium. Data on $\mathrm{ICL}$ are shown in panels A-D, and data on strand breaks in E-H. For CEES, each column represents mean values + SEM from 3 independent experiments $(n=3)$ and for all other agents at least 7 independent experiments $(n=7)$, with 4 replicates each. Data sets were compared using 1 -way ANOVA and Dunnett's Multiple Comparison Post Test $\left({ }^{*} P<0.05 ;{ }^{* * *} P<0.001\right)$.

DNA damage within the same experiment. This does not lengthen the procedure or increase expenses. Among the crosslinking agents tested, only melphalan led to formation of DNA strand breaks, as did the monofunctional agent CEES (Fig. 1, right panel). The melphalan-induced breaks might represent intermediates of nucleotide-excision or crosslink repair. However, at the later time point of $14 \mathrm{~h}$ after treatment apoptotic DNA cleavage cannot be excluded. Mitomycin C and SM did not induce any detectable DNA strand breaks, and the amount of double stranded DNA remained at control level. This led us to assume that the reverse FADU assay 


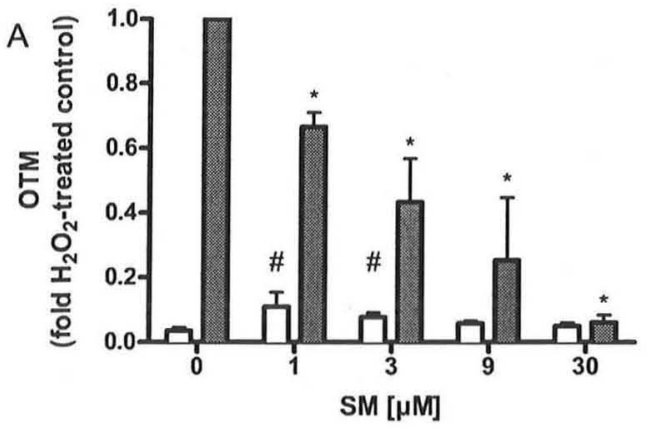

OTM

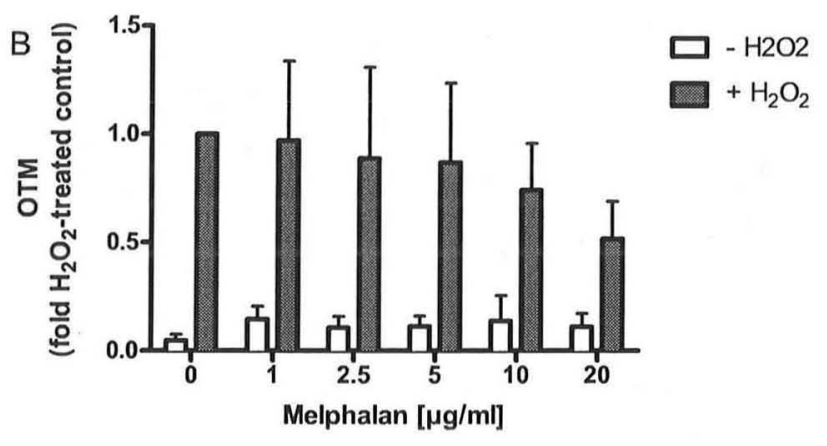

Fig. 2. Frequency of DNA crosslinks and DNA strand breaks in human PBMC after treatment with sulphur mustard or melphalan and analyzed by comet assay. PBMC were treated with SM ( 30 min; panel A) or melphalan (60 min; panel B). Medium containing mutagen was removed; cells were washed with PBS and resuspended in fresh culture medium. After treatment with melphalan cells were allowed to recover for $14 \mathrm{~h}$. Each column represents olive tail moment (OTM) mean values \pm SD normalized to OTM of $\mathrm{H}_{2} \mathrm{O}_{2}$ treated controls of 4 independent experiments $(n=4)$. In each experiment 100 cells were scored. White columns, DNA strand breaks; black columns, ICL. Each data set was compared to control using 1-way ANOVA with Dunnett's Post Test ("significant vs. $\mathrm{H}_{2} \mathrm{O}_{2}$ control, \#significant vs. untreated control, $P<0.05)$.

is not influenced by putative protein-DNA crosslinks. Otherwise a dose-dependent increase in double-stranded DNA over the control level in the direct FADU assay might be expected.

\subsection{Direct comparison of FADU assay with reversed comet assay}

To directly compare the sensitivity of the reverse FADU assay with previously described methods for ICL detection, we performed the reverse comet assay (Fig. 2). The reverse comet assay is currently the most frequently used method for the detection of DNA crosslinks. PBMC of different donors were treated with melphalan and SM as described above for the reverse FADU assay. The detection of DNA crosslinks was based on reduction of olive tail moment (OTM) by the agents after induction of strand breaks with high dose of hydrogen peroxide. Our data show that the sensitivity of comet assay for detection of DNA crosslinks was comparable to that of the automated FADU assay. DNA crosslinks were detected in human PBMC after treatment with $1 \mu \mathrm{M}$ SM $(P<0.05$, Dunnett's Multiple Comparison Post Test) (Fig. 2). On the other hand, the DNA crosslinks detected in melphalan-treated PBMC did not reach statistical significance. The induction of DNA crosslinks by either compound was dose-dependent, as was the case with the reverse FADU assay. In contrast, strand break induction was elevated after treatment with the agents but this did not correlate with the dose applied.

\subsection{Impact of proliferation on results from reversed FADU assay}

In the experiments described above, we have shown that the reverse FADU assay is suitable for the assessment of DNA interstrand crosslinks in human PBMC. The next question we addressed was if the FADU assay can also be applied for proliferating cells. Furthermore, we wanted to know if not only induction of crosslinks but also repair processes could be monitored by FADU. Thus, DNA crosslinks and strand breaks were followed up in time-course experiments after treatment with $10 \mu \mathrm{g} / \mathrm{ml}$ melphalan in resting human PBMC and proliferating human T lymphocytes (Fig. 3). Our data show that the acquisition of DNA damage was not impaired by proliferation. As a matter of fact, the induction of crosslinks and their repair was even more pronounced in proliferating lymphocytes than in PBMC. This might be due to relatively higher amount of euchromatin in proliferating cells compared to $\mathrm{G}_{0}$ cells or higher cellular uptake of the drug. The incorporation of crosslinks in proliferating cells was observed already within the first hour after treatment and reached a maximum by $2 \mathrm{~h}$ after treatment, while in resting cells at this time point crosslink induction was noticed and only increased with time to reach a maximum by $14 \mathrm{~h}$ after treatment. Within both cell types the repair process was observed starting at $18 \mathrm{~h}$ after treatment. Once again DNA repair was more effective in proliferating cells. Interestingly strand break induction appeared to be independent of proliferation status and was observed $14 \mathrm{~h}$ after treatment. Our data reveal that the reverse FADU assay detects with high sensitivity DNA crosslinks both in resting and proliferating cells. It is also a convenient method for assessment of DNA repair and differences in DNA damage different cell types.

\subsection{Detection of crosslinks in whole blood samples}

As the reverse FADU assay was primarily developed for highthroughput applications such as epidemiological and clinical studies, it was important to determine the minimal sample volume necessary and to define optimal conditions for storage and shipment of samples between laboratories. Especially in case of cancer patients, there is a strong pressure to minimize the volume of blood to be sampled, in order to avoid the risk of compromising the patient's health even further. A great reduction in the blood volume required was achieved by using a protocol of erythrocyte lysis instead of density gradient centrifugation, and as little as $100 \mu \mathrm{l}$ of blood proved sufficient for the analysis (Fig. 4). Comparison of human leukocyte preparation with PBMC showed that there was no difference in the detection of DNA crosslinks or in DNA strand breaks upon melphalan treatment of the cells ex vivo.

\subsection{Assessment of DNA crosslinks by FADU assay in cryopreserved samples}

In the context of epidemiological studies, sample collection often takes place at distant geographic locations, thus necessitating implementation of appropriate cell preservation and shipment protocols. To check if cryopreservation of the samples has an impact on the results from the reverse FADU, we compared the crosslinks level in human PBMC $14 \mathrm{~h}$ after treatment with melphalan processed immediately with samples that were cryopreserved by standard cell culture procedure and stored in liquid nitrogen for one month (Fig. 5). Our data revealed that cryopreservation and liquid nitrogen storage had no influence on the detection of DNA crosslinks. However, the measurement of DNA strand breaks could not be performed reliably with thawed samples. The thawing process was critical for cells, and we observed that additional breaks appeared, which led to overestimation of DNA damage in some cases and to great variation between experiments. 

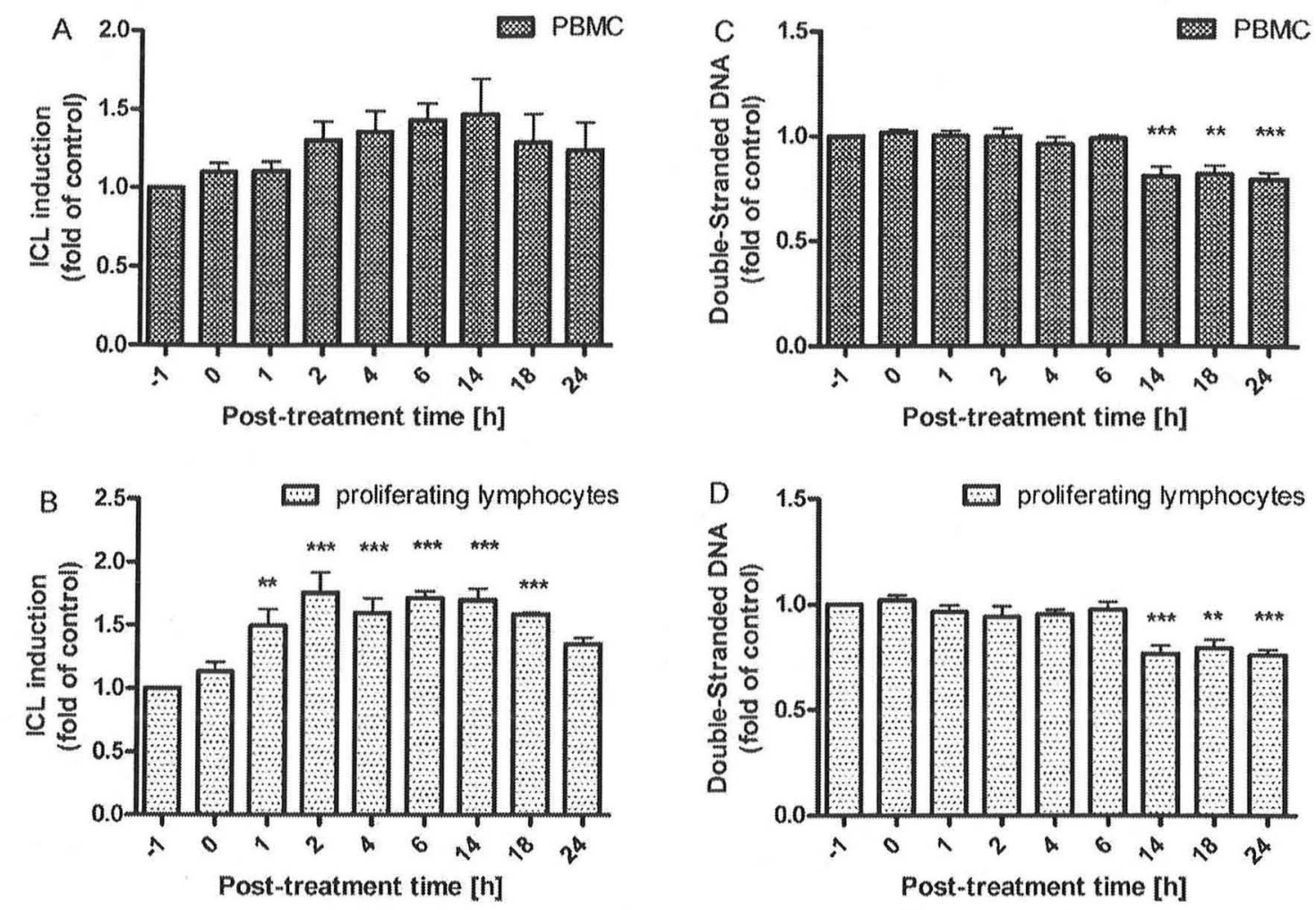

Fig. 3. Frequency of ICL and DNA strand breaks as a function of time after treatment with $10 \mu \mathrm{g} / \mathrm{ml}$ melphalan in human PBMC and proliferating human lymphocytes, respectively, as analyzed by FADU assay. Cells were treated with $10 \mu \mathrm{g} / \mathrm{ml}$ melphalan for 60 min. Cells were washed with PBS, resuspended in fresh medium and cultivated for the time period indicated. FADU assay was performed by the standard procedure. Data on ICL are shown in panels (A) and (B), and data on strand breaks in (C) and (D), In order to bring lymphocytes to proliferation, PBMC were incubated with $5 \mu \mathrm{g} / \mathrm{ml}$ phytohemagglutinin-L (PHA-L) for $48 \mathrm{~h}$ before treatment. PHA-L was present in medium during whole duration of the experiment. Data on PBMC are shown in panels (A) and (C), and data on proliferating human lymphocytes in (B) and (D). Each column represents mean values \pm SEM from at least 4 independent experiments $(n=4)$. Data sets were compared using 1 -way ANOVA with Dunnett's Multiple Comparison Post Test ( $* *<0.01$; $\left.{ }^{* * *} P<0.001\right)$.

\subsection{Validation of FADU assay with coded samples}

All the above data support the idea that the (reverse) FADU assay is a suitable candidate for high-throughput analysis, e.g. in the context of epidemiological studies. For final validation of the assay, we assumed a hypothetical scenario where victims of SM exposure should be identified. For this purpose human PBMC were treated $e x$ vivo with different concentrations of SM, cryopreserved, shipped to the analytical lab and encoded. The SM concentrations used were below $100 \mu \mathrm{M}$, thus mimicking in vivo exposure levels that would not necessarily lead to characteristic acute symptoms including skin blisters. The samples were analyzed by reverse FADU assay and data were evaluated statistically by comparison with a previously established concentration-response curve (Fig. 1). After breaking the code, our data showed that we have been able to distinguish samples exposed to doses higher or equal to our detection limit of $3 \mu \mathrm{M}$ ("victims") from controls with $100 \%$ accuracy (Table 1). In some cases the SM concentration was underestimated but alto-
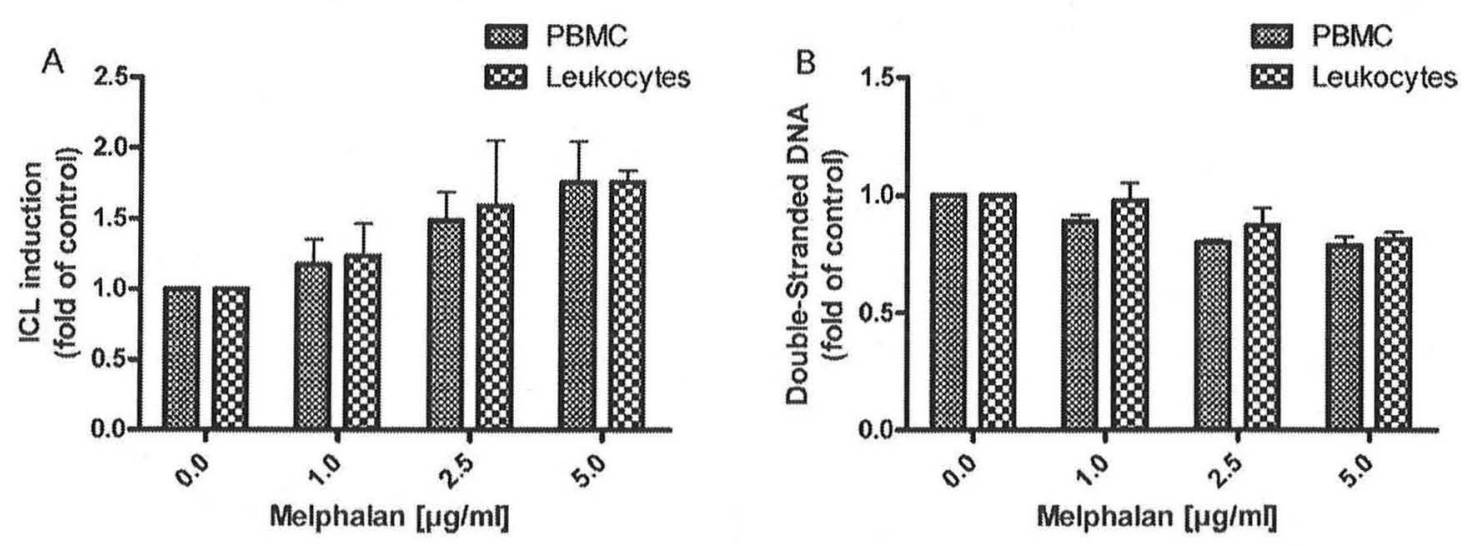

Fig. 4. Frequency of ICL (A) and DNA strand breaks (B) in human PBMC and human leukocytes after treatment with melphalan analyzed by FADU assay. PBMCs and freshly collected whole blood samples from the same donor were treated with melphalan for $14 \mathrm{~h}$. Leukocytes were isolated by the erythrocyte lysis method and analyzed simultaneously with PBMC by FADU assay. Data on ICL are shown in panels A, and data on DNA strand breaks in panel B. Each column represents mean values \pm SEM from 4 independent experiments with 4 replicates $(n=4)$. Data sets were compared using 2-way ANOVA $(P<0.05)$. 


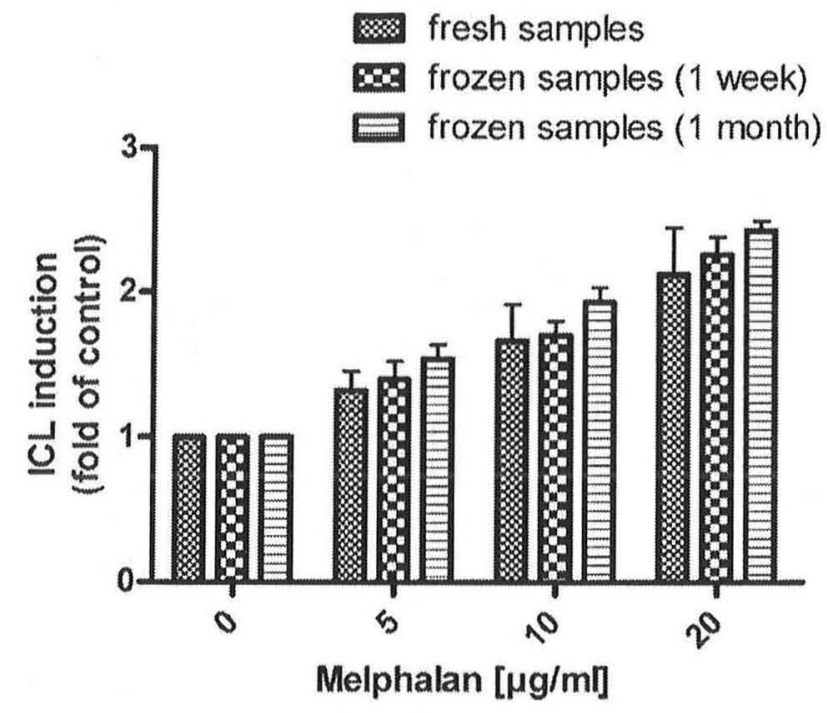

Fig. 5. Frequency of ICL after melphalan treatment in human PBMC directly analyzed by FADU assay and after cryopreservation. PBMC were treated with melphalan for 60 min, washed with PBS and allowed to recover in fresh medium for $14 \mathrm{~h}$. PBMC were harvested and split into three aliquots. Aliquot 1 was analyzed directly by FADU assay. Aliquots 2 and 3 were cryopreserved and analyzed by FADU assay after storage in $-80^{\circ} \mathrm{C}$ for 1 week and 1 month, respectively. Each column represents mean values \pm SEM from 4 independent experiments with 4 replicates $(n=4)$. Data sets were compared using 2-way ANOVA $(P<0.05)$.

\section{Table 1}

Analysis of ICL by FADU assay in coded samples. PBMC were treated with SM at various concentrations, cryopreserved and shipped from one laboratory (in Munich, Germany) to the other (in Konstanz, Germany). PBMC were analyzed by FADU assay in a blinded fashion and statistical evaluation was done by one-sample $t$-test to a dose response curve previously established for SM. Then the code was broken and the predicted exposure was compared with real exposure.

\begin{tabular}{rllc}
\hline Sample code & $\begin{array}{l}\text { Relative } \\
\text { fluorescence } \\
\text { signal }(\%)\end{array}$ & $\begin{array}{l}\text { Predicted exposure } \\
\text { SM }(\mu \mathrm{M})\end{array}$ & $\begin{array}{l}\text { Real exposure SM } \\
(\mu \mathrm{M})\end{array}$ \\
\hline 1 & 22.18 & 0 & 0 \\
2 & 52.20 & 9.5 & 30 \\
3 & 21.84 & 0 & 0 \\
4 & 36.36 & 3 & 3 \\
5 & 13.03 & 0 & 1 \\
6 & 15.06 & 0 & 0.3 \\
7 & 13.03 & 0 & 0 \\
8 & 32.25 & 1 & 3 \\
9 & 56.65 & 9.5 & 10 \\
10 & 69.01 & 30 & 30 \\
11 & 68.00 & 30 & 30 \\
12 & 33.84 & $1-3$ & 3 \\
13 & 14.02 & 0 & 0.3 \\
14 & 62.67 & 30 & 9.5 \\
15 & 24.17 & $0-1$ & 1 \\
16 & 16.61 & 0 & 0 \\
\hline
\end{tabular}

gether our study proves the high accuracy and reproducibility of FADU data.

\section{Discussion}

The present paper is the first description of the use of the reverse FADU assay for the assessment of DNA interstrand crosslinks. The assay was previously applied for detection of DNA strand breaks but despite many advantages it has never reached the same popularity as the comet assay. The comet assay and also alkaline elution, however, are methods that are time-consuming and allow processing of only a limited sample number. The assessment of ICL would be of particular interest in cancer therapy and in personalized risk assessment, where standardized, high-throughput assay would be preferred. For this purpose we developed the reverse FADU assay. The assay is fast, easy, fully automated, highly reproducible and of low cost. All these advantages make the reverse FADU an attractive candidate for large monitoring studies.

In the course of the present study, we optimized the assay conditions towards the highest resolution for the detection of ICL. The number of X-ray-induced DNA strand breaks to be administered in the reverse assay as well as unwinding conditions were systematically investigated and optimized resulting in development of the present protocol.

We were able to detect ICL in human PBMC after treatment with SM and two cytostatic drugs, i.e. mitomycin C and melphalan. SM was initially developed as a chemical warfare agent. Its last military use was during the Iraq-Iran war in the 1980 s. However, accidental exposure of civilians is being reported even today, from the inappropriate disposal of sea-dumped ammunition or old depots. SM is a documented mutagen and carcinogen, and an increased tumour risk is caused even by low-dose exposure that does not evoke characteristic skin lesions (Balali-Mood and Hefazi, 2006). Biomonitoring based on assessment of ICL is, therefore, an attractive option. This prompted us to include SM in the present study along with anticancer drugs. For all three substances we were able to detect ICL in a dose dependent manner. The dose response was assessed at the time point of maximal crosslinks induction for the respective substance, which we determined previously in time-course experiments. ICL were equally well detected independent on chemical structure. The assessment of ICL was possible for $5 \mu \mathrm{g} / \mathrm{ml}$ mitomycin $\mathrm{C}$ and melphalan, respectively, and $3 \mu \mathrm{M} \mathrm{SM}$. Based on the quantitative data available for SM (Mol et al., 1993), we estimated that the reverse FADU assay allows detection of $1 \mathrm{ICL}$ per $4.2 \times 10^{6} \mathrm{bp}$, equivalent to approximately 780 crosslinks per genome.

The main question remains the sensitivity of the reverse FADU assay in comparison to other methods. The closest competitor is obviously the reverse comet assay. In our hands the sensitivity of the comet assay was comparable to that of the FADU assay. The minimal concentration for damage induction detectable by comet assay was $1 \mu \mathrm{M}$ SM. Melphalan induced crosslinks, however, were not detectable in the concentration range investigated. A similar relationship in sensitivity was obtained for comparison of FADU $(15 \mu \mathrm{M})$ with published data for mitomycin $\mathrm{C}(10 \mu \mathrm{M})$ (Merk and Speit, 1999). A major advantage of the reverse FADU assay over the comet assay is the absolutely stringent standardization of all steps in the protocol and read-out. Thus, our inter-assay variability is negligible and we expect the same to be the case for inter-laboratory variability. Due to the easy and fast procedure, which can be completed in $3 \mathrm{~h}$, and the microtiter format used, the assay has a high throughput. It should therefore be very suitable for large cohort studies. Its applicability for clinical studies is currently under investigation. Furthermore, the reverse FADU assay is very promising for the biomonitoring of genotoxic exposure. Last but not least, in our hands the assay proved to be a useful tool for assessment of DNA damage and repair in cell culture experiments aimed at elucidation of molecular mechanisms.

\section{Funding}

This work was supported by the German Bundesamt für Wehrtechnik und Beschaffung, Grant U2.3 E/UR3G/5G121/5A805.

\section{Conflict of interest statement}

None declared. 


\section{Acknowledgements}

We thank Ms. Katharina Hüttner, Mrs. Elisabeth Müßig, Mr.Steffen Müller, Mr. Ram Naresh Prasad and Ms. Cornelia Wollner for their excellent technical assistance.

\section{References}

Balali-Mood, M., Hefazi, M., 2006. Comparison of early and late toxic effects of sulfur mustard in Iranian veterans. Basic Clin. Pharmacol. Toxicol. 99, 273-282.

Baumstark-Khan, C., Griesenbach, U., Rink, H., 1992. Comparison of DNA strand break induction in $\mathrm{CHO}$ cells measured by alkaline elution and by fluorometric analysis of DNA unwinding (FADU). Free Radic. Res. Commun. 16, 381-389.

Baumstark-Khan, C., Hentschel, U., Nikandrova, Y., Krug, J., Horneck, G., 2000. Fluorometric analysis of DNA unwinding (FADU) as a method for detecting repair-induced DNA strand breaks in UV-irradiated mammalian cells. J. Photochem. Photobiol. 72, 477-484.

Birnboim, H.C., Jevcak, J.J., 1981. Fluorometric method for rapid detection of DNA strand breaks in human white blood cells produced by low doses of radiation. Cancer Res. 41, 1889-1892.

Brendler-Schwaab, S. Hartmann, A., Pfuhler, S., Speit, G, 2005. The in vivo comet assay: use and status in genotoxicity testing. Mutagenesis 20, 245 254.

Colvin, M.E., Quong.J.N., 2002. DNA-alkylating events associated with nitrogen mustard based anticancer drugs and the metabolic byproduct Acrolein. Adv. DNA Seq-Spec. Agents 4, 29-46.

Dimopoulos, M. A Souliotis, V. L, Anagnostopoulos, A, Bamia, C, Pouli, A, Baltadakis, I., Terpos, E., Kyrtopoulos, S.A., Sfikakis, P.P., 2007. Melphalan-induced DNA damage in vitro as a predictor for clinical outcome in multiple myeloma. Haematologica 92, 1505-1512.

Dronkert, M.L., Kanaar, R., 2001. Repair of DNA interstrand cross-links. Mutat. Res. $486,217-247$.

Forchhammer, L., Brauner, E.V., Folkmann, J.K., Danielsen, P.H., Nielsen, C., Jensen, A. Loft, S. Friis, G., Moller, P., 2008. Variation in assessment of oxidatively damaged DNA in mononuclear blood cells by the comet assay with visual scoring. Mutagenesis 23, 223-231
Hartley, J.M., Spanswick, V.J., Gander, M., Giacomini, G., Whelan, J., Souhami, R.L. Hartley, J.A., 1999. Measurement of DNA cross-linking in patients on ifosfamide therapy using the single cell gel electrophoresis (comet) assay. Clin. Cancer Res. 5, 507-512.

Kee, Y., D'Andrea, A.D., 2010. Expanded roles of the Fanconi anemia pathway in preserving genomic stability. Genes Dev. 24, 1680-1694.

Lawley, P.D., Phillips, D.H., 1996. DNA adducts from chemotherapeutic agents. Mutat. Res. 355, 13-40.

Merk, O., Speit, G., 1999. Detection of crosslinks with the comet assay in relationship to genotoxicity and cytotoxicity. Environ. Mol. Mutagen. 33, 167-172.

McHugh, P.J., Spanswick, V.J., Hartley, J.A., 2001. Repair of DNA interstrand crosslinks: molecular mechanisms and clinical relevance. Lancet Oncol. 2. 483-490.

Mol, M.A., van der Schans, G.P., Lohman, P.H., 1993. Quantification of sulfur mustard induced DNA interstrand cross-links and single-strand breaks in cultured human epidermal keratinocytes. Mutat. Res. 294, 235-245.

Moreno-Villanueva, M., Pfeiffer, R., Sindlinger, T., Leake, A., Muller, M., Kirkwood, T.B., Burkle, A., 2009. A modified and automated version of the 'Fluorimetric Detection of Alkaline DNA Unwinding' method to quantify formation and repair of DNA strand breaks. BMC Biotechnol. 9, 39.

Noll, D.M., Mason, T.M., Miller, P.S., 2006. Formation and repair of interstrand crosslinks in DNA. Chem. Rev. 106, 277-301.

Pfuhler, S., Wolf, H.U., 1996. Detection of DNA-crosslinking agents with the alkaline comet assay. Environ. Mol. Mutagen. 27, 196-201.

Roos, W.P., Binder, A., Bohm, L., 2000. Determination of the initial DNA damage and residual DNA damage remaining after $12 \mathrm{~h}$ of repair in eleven cell lines at low doses of irradiation. Int. J. Radiat. Biol. 76, 1493-1500.

Roos, W., Baumgartner, M., Kaina, B., 2004. Apoptosis triggered by DNA damage O6methylguanine in human lymphocytes requires DNA replication and is mediated by $\mathrm{p} 53$ and Fas/CD95/Apo-1. Oncogene 23, 359-367.

Souliotis, V.L. Dimopoulos, M.A., Sfikakis, P.P., 2003. Gene-specific formation and repair of DNA monoadducts and interstrand cross-links after therapeutic exposure to nitrogen mustards. Clin. Cancer Res. 9, 4465-4474

Spanswick, V.J., Craddock, C., Sekhar, M., Mahendra, P., Shankaranarayana, P. Hughes, R.G., Hochhauser, D., Hartley, J.A., 2002. Repair of DNA interstrand crosslinks as a mechanism of clinical resistance to melphalan in multiple myeloma. Blood 100, 224-229. 